\title{
Precision Control on Development Design of the Injection Plastic Part based on Reverse Engineering
}

\author{
Jun Fan ${ }^{1, a}$, Leigang Wang ${ }^{2}$ \\ 1 Suqian College, $\mathrm{CHN}$ \\ 2 Jiangsu University, CHN
}

\begin{abstract}
Product development design needs to go through four phases. These phases are construction phase, development phase, emulation phase and assessment phase. The deviation composition of these phases is studied. In the process of reverse development design, emphasizing on phase deviation, taking the injection plastic part for example, the quantitative precision control on the process is satisfied effectively.
\end{abstract}

\section{Introduction}

In the development process of products[1-5], the precision analysis is always a crucial technical problem. With reverse engineering technology used in product development increasingly, accuracy control becomes a core technology issue[6-8]. In this paper, a quantitative analysis on deviation distribution of development design and reverse development design is implemented, at the same time, precision control on reverse development design of the injection plastic part is realized.

\section{Deviation Distribution of Development Design}

\subsection{Phase Analysis}

Product development design needs to go through four phases. These phases are construction phase, development phase, emulation phase and assessment phase. The phases of development design process is shown as Figure 1. The construction phase includes reconstruction process and appraisal process. The construction phase is shown as Figure 2. The development phase includes analysis process and optimization process. The development phase is shown as Figure 3. The emulation phase includes static process and dynamic process. The emulation phase is shown as Figure 4. The assessment phase includes product process and assembly process. The assessment phase is shown as Figure 5.

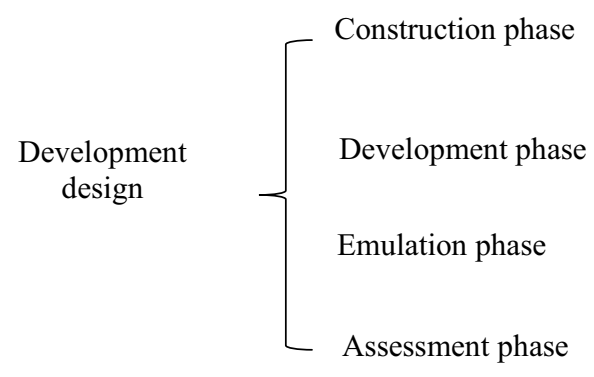

Figure 1. Development design.



Figure 2. Construction phase.

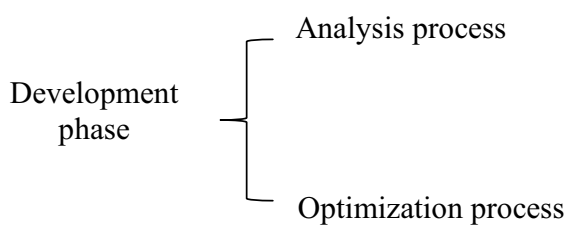

Figure 3. Development phase.

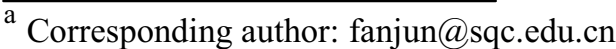




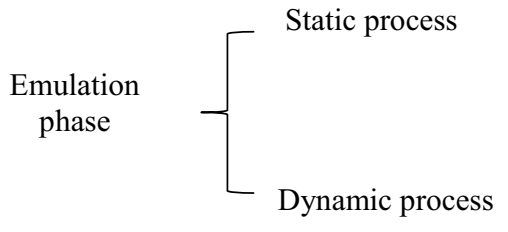

Figure 4. Emulation phase.

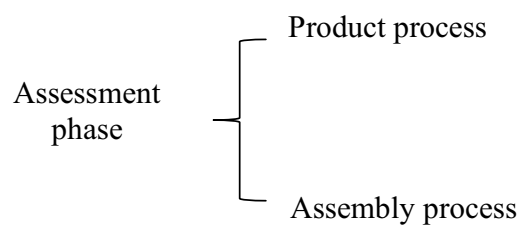

Figure 5. Assessment phase.

\subsection{Deviation Composition}

Based on the phase analysis, deviation of development design is made primarily from construction deviation, development deviation, emulation deviation and assessment deviation. The deviation of development design is shown as Figure 6. The construction deviation involves reconstruction deviation and appraisal deviation. The construction deviation is shown as Figure 7. The development deviation involves analysis deviation and optimization deviation. The development deviation is shown as Figure 8. The emulation deviation involves static deviation and dynamic deviation. The emulation deviation is shown as Figure 9.The assessment deviation involves product deviation and assembly deviation. The assessment deviation is shown as Figure 10.

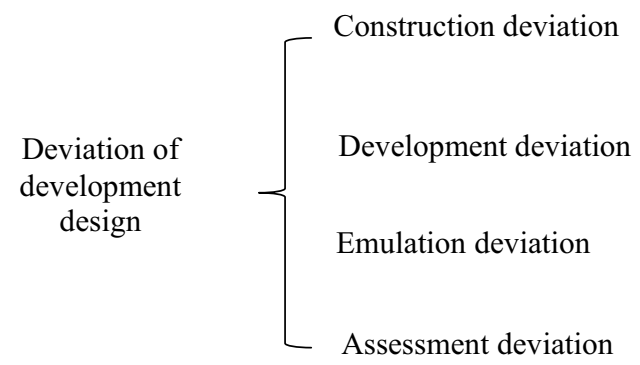

Figure 6. Deviation of development design.

According to Figure 6, the overall deviation formula of the development design is presented in Equation1. $\Delta{ }_{\text {development design }}^{2}$
$\Delta_{\text {construction }}^{2}+\Delta_{\text {development }}^{2}+\Delta_{\text {emulation }}^{2}+$
${ }_{\text {assesment }}^{2}$

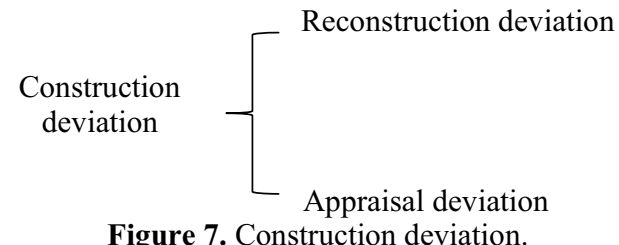

Figure 7. Construction deviation.
In Equation 1, according to Figure 7, the construction deviation formula is presented in Equation 2.

$$
\Delta_{\text {construction }}^{2}=\Delta_{\text {reconstruction }}^{2}+\Delta_{\text {appraisal }}^{2}
$$

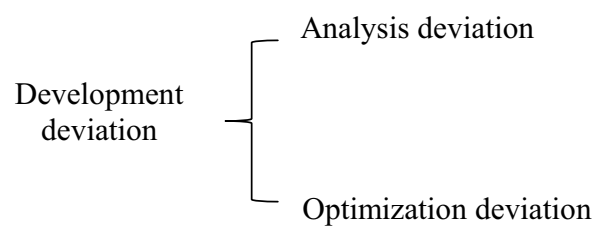

Figure 8. Development deviation.

In Equation 1, according to Figure 8, the development deviation formula is presented in Equation 3.

$$
\begin{gathered}
\Delta_{\text {development }}^{2}=\Delta{ }_{\text {analysis }}^{2}+\Delta{ }_{\text {optimization }}^{2} \\
\text { deviation } \\
\text { Static deviation } \\
\text { Dynamic deviation }
\end{gathered}
$$

Figure 9. Emulation deviation.

In Equation 1, according to Figure 9, the emulation deviation formula is presented in Equation 4.

$$
\Delta_{\text {emulation }}^{2}=\Delta_{\text {static }}^{2}+\Delta_{\text {dynamic }}^{2}
$$

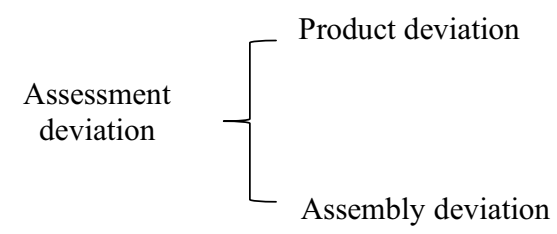

Figure 10. Assessment deviation.

In Equation 1, according to Figure 10, the assessment deviation formula is presented in Equation 5.

$$
\Delta_{\text {assessment }}^{2}=\Delta_{\text {product }}^{2}+\Delta_{\text {assembly }}^{2}
$$

\section{Accuracy Study on Reverse Development Design}

\subsection{Phase Analysis}

There are four phases during reverse development design, namely, reverse phase, reverse development phase, reverse emulation phase and reverse assessment phase. The phases of reverse development design is shown as Figure 11. The reverse phase includes rebuilding process and appraisal process. The reverse phase is shown as Figure 12. The rebuilding process covers data process and prototype process. The rebuilding process is shown as Figure 13. The reverse development phase includes reverse analysis process and reverse optimization process. The reverse development phase is shown as Figure 14. The reverse emulation phase includes reverse static process and reverse dynamic process. The reverse emulation phase is shown as Figure 15. The reverse 
assessment phase includes reverse product process and reverse assembly process. The reverse assessment phase is shown as Figure 16.

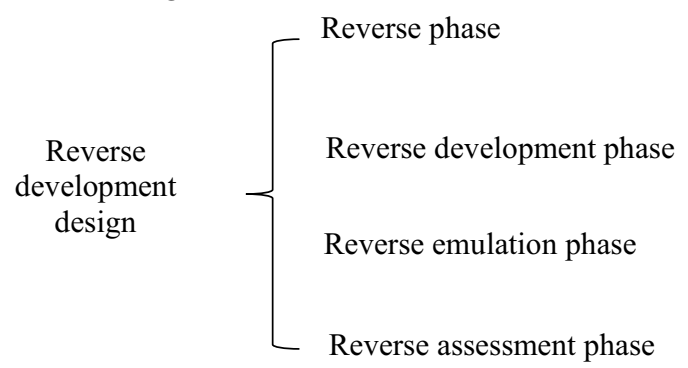

Figure 11. Reverse development design.

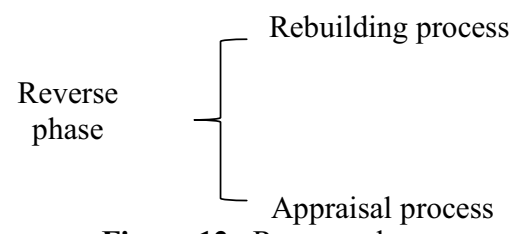

Figure 12. Reverse phase.

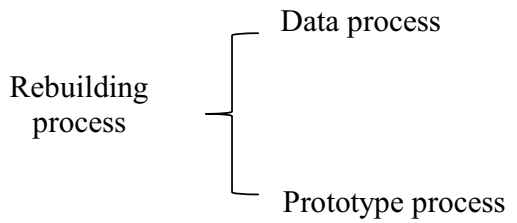

Figure 13. Rebuilding process.

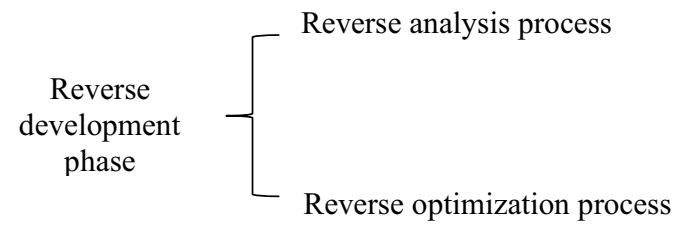

Figure 14. Reverse development phase.

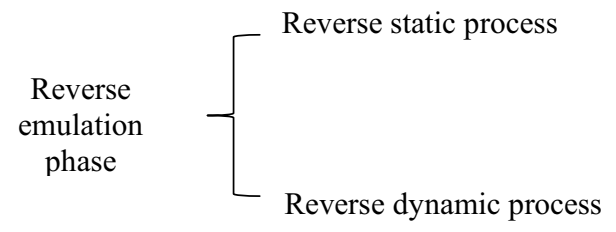

Figure 15. Reverse emulation phase.

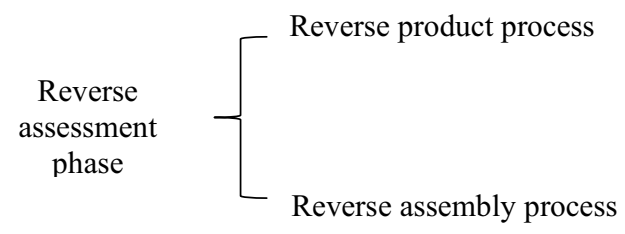

Figure 16. Reverse assessment phase.

\subsection{Deviation Research}

Based on the phase analysis of reverse development design, deviation of whole process involves reverse deviation, reverse development deviation, reverse emulation deviation and reverse assessment deviation. The deviation of reverse development design is shown as Figure 17. The reverse deviation covers rebuilding deviation and appraisal deviation. The reverse deviation is shown as Figure 18. The rebuilding deviation incorporates data deviation and prototype deviation. The rebuilding deviation is shown as Figure 19. The reverse development deviation covers reverse analysis deviation and reverse optimization deviation. The reverse development deviation is shown as Figure 20. The reverse emulation deviation covers reverse static deviation and reverse dynamic deviation. The reverse emulation deviation is shown as Figure 21. The reverse assessment deviation covers reverse product deviation and reverse assembly deviation. The reverse assessment deviation is shown as Figure 22.

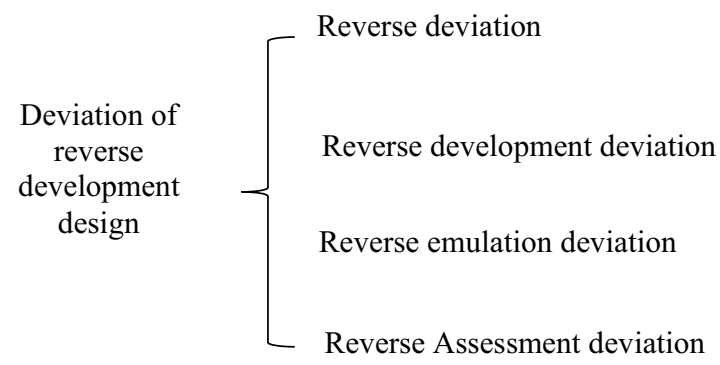

Figure 17. Deviation of reverse development design.

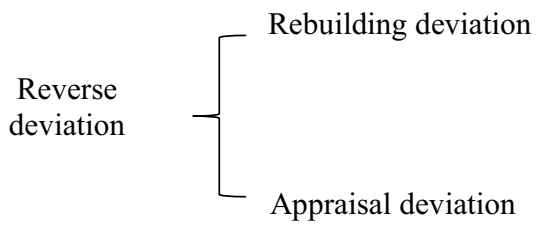

Figure 18. Reverse deviation.

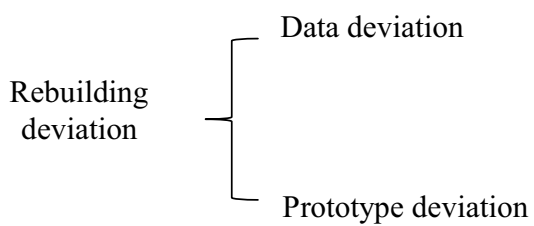

Figure 19. Rebuilding deviation.

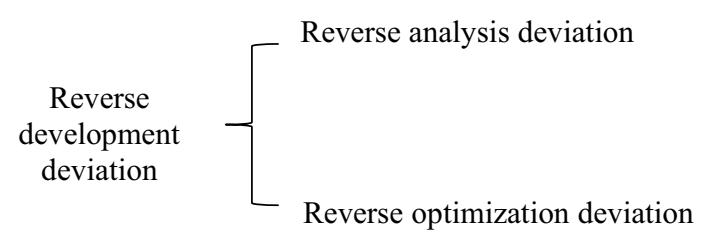

Figure 20. Reverse development deviation. 


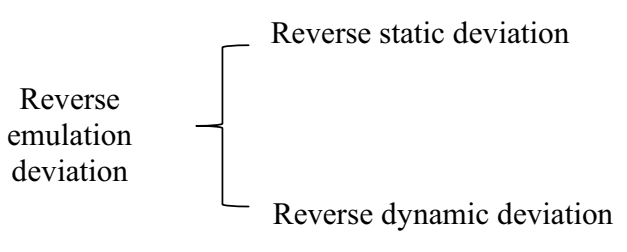

Figure 21. Reverse emulation deviation.

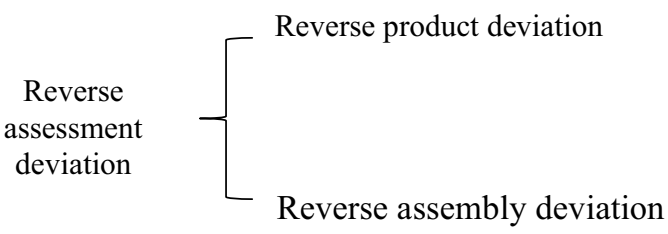

Figure 22. Reverse assessment deviation.

On the basis of the whole deviation analysis, the overall deviation formula of the process is presented in Equation 6.

$$
\begin{aligned}
& \Delta{ }_{\text {reverse development design }}^{2}=\Delta{ }_{\text {reverse }}^{2}+\Delta_{2}^{2} \text { reverse development } \\
& \Delta \Delta_{\text {reverse emulation }}^{2}+\Delta^{2} \text { reverse assessment }
\end{aligned}
$$

In Equation 6, the reverse deviation formula is presented in Equation 7.

$$
\Delta{ }^{2} \text { reverse }=\Delta{ }_{\text {rebuilding }}^{2}+\Delta^{2} \text { appraisal }
$$

In Equation 7, the rebuilding deviation formula is presented in Equation 8.

$$
\Delta{ }_{\text {rebuilding }}^{2}=\Delta{ }_{\text {data }}^{2}+\Delta{ }_{\text {prototype }}
$$

In Equation 6, the reverse development deviation formula is presented in Equation 9.

$\Delta{ }^{2}$ reverse development $=\Delta{ }^{2}$ reverse analysis $+\Delta^{2}$ rever

In Equation 6, the reverse emulation deviation formula is presented in Equation 10.

$$
\Delta_{\text {reverse emulation }}^{2}=\Delta_{\text {reverse static }}^{2}+\Delta^{2}{ }_{\text {reverse dynamic }}
$$

In Equation 6, the reverse assessment deviation formula is presented in Equation 11.
$\Delta^{2}$

(11)

\section{Instance}

Point data of a injection plastic part is shown as Figure 23. The deviation of reverse development design is limited within $0.1 \mathrm{~mm}$.

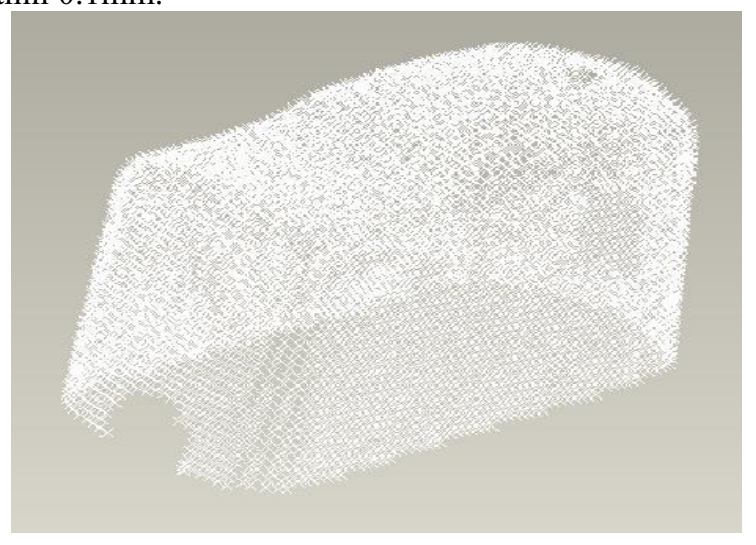

Figure 23. Point data.

The reverse deviation is restricted in about $0.08 \mathrm{~mm}$. The reverse development deviation is restricted in about $0.01 \mathrm{~mm}$. The reverse static emulation is shown in Figure 24. Generally speaking, the reverse emulation deviation and the reverse assessment deviation are restricted in about $0.02 \mathrm{~mm}$. On the basis of Equation 6, the overall deviation of the reverse development design is $0.085 \mathrm{~mm}$, which satisfies the deviation need of less than $0.10 \mathrm{~mm}$. Therefore, the precision control of the reverse develonment decion is raslized

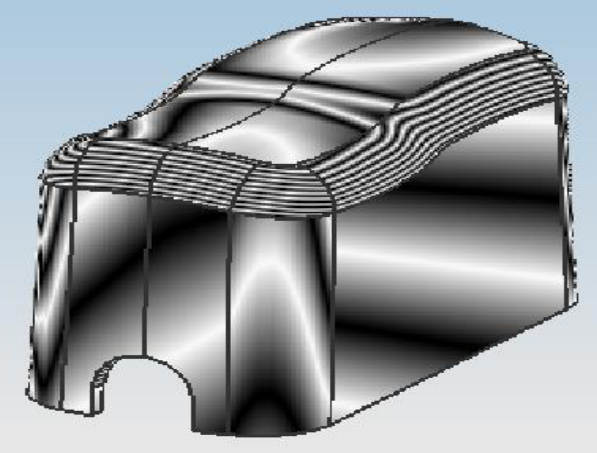

Figure 24. Reverse static emulation.

\section{Conclusions}

In this paper, precision on development design of the injection plastic part based on reverse engineering is under control. Analysis on the deviation composition of reverse development design is very significant for accuracy control.

\section{Acknowledgements}

This study was funded by the Scientific Research Project of Suqian College(2015KY30), the Jiangsu Brand Professional Construction Engineering Project in Colleges and Universities(PPZY2015C252), the Student Innovation Training Project of Suqian College in 2015 .

\section{References}

1. Xiaobo Shi.Manufacturing Automation.32,122 (2010)

2. Qiang Huang. Coal Mine Machinery. 32,12 (2011)

3. Xuehui Chen, Lei Huang, Jingfa Lei, et al. Coal Mine Machinery. 32,57 (2011)

4. Yuan Wang, Jianguang Fang, Jingren Wang, et al. Automotive Engineering. 34,847 (2012)

5. Shuhong Ge, Ding Fan, Kai Peng. Computer Simulation. 29,81 (2012)

6. Jun Fan, Leigang Wang. Manufacturing Automation. 35,131 (2013)

7. Jun Fan, Leigang Wang. Applied Mechanics and Materials,538,83(2014)

8. Xiaogang ji, Guangrong Gong. Mechanical Science and Technology. 25,322 (2006) 